\title{
Carrier-envelope frequency stabilization of a Ti:sapphire oscillator using different pump lasers
}

\author{
Andreas Vernaleken, ${ }^{1, *}$ Bernhard Schmidt, ${ }^{2}$ Martin Wolferstetter, ${ }^{2}$ \\ Theodor W. Hänsch, ${ }^{1}$ Ronald Holzwarth, ${ }^{1,2}$ and Peter Hommelhoff ${ }^{1}$ \\ ${ }^{1}$ Max-Planck-Institut für Quantenoptik, Hans-Kopfermann-Str. 1, 85748 Garching, Germany \\ ${ }^{2}$ Menlo Systems GmbH, Am Klopferspitz 19a, 82152 Martinsried, Germany \\ *andreas.vernaleken@mpq.mpg.de
}

\begin{abstract}
We investigate the suitability of various commercially available pump lasers for operation with a carrier-envelope offset frequency stabilized Ti:sapphire oscillator. Although the tested pump lasers differ in their setup and properties (e.g., single vs. multi-mode), we find that they are all well-suited for the purpose. The residual rms phase noise (integrated between $20 \mathrm{~Hz}$ and $5 \mathrm{MHz}$ ) of the stabilized oscillator is found to be below $160 \mathrm{mrad}$ with each pump laser, corresponding to less than $1 / 40$ of an optical cycle. Differences in performance vary slightly. In particular, our results indicate that the latest generation of multi-mode pump lasers can be used for applications where precise phase control of the oscillator is strictly required.
\end{abstract}

(C) 2012 Optical Society of America

OCIS codes: (120.5050) Phase measurement; (140.3425) Laser stabilization; (140.5560) Pumping; (140.7090) Ultrafast lasers; (320.7160) Ultrafast technology.

\section{References and links}

1. Th. Udem, R. Holzwarth, and T. W. Hänsch, "Optical frequency metrology," Nature 416, 233-237 (2002).

2. S. A. Diddams, Th. Udem, J. C. Bergquist, E. A. Curtis, R. E. Drullinger, L. Hollberg, W. M. Itano, W. D. Lee, C. W. Oates, K. R. Vogel, and D. J. Wineland, “An optical clock based on a single trapped ${ }^{199} \mathrm{Hg}^{+}$ion," Science 293, 825-828 (2001).

3. P. B. Corkum and F. Krausz, "Attosecond science," Nat. Phys. 3, 381-387 (2007).

4. F. Krausz and M. Ivanov, "Attosecond physics," Rev. Mod. Phys. 81, 163-234 (2009).

5. A. Bartels, D. Heinecke, and S. A. Diddams, "10-GHz self-referenced optical frequency comb," Science 326, 681 (2009)

6. O. Mücke, R. Ell, A. Winter, J. Kim, J. Birge, L. Matos, and F. Kärtner, "Self-referenced $200 \mathrm{MHz}$ octavespanning Ti:sapphire laser with 50 attosecond carrier-envelope phase jitter," Opt. Express 13, 5163-5169 (2005).

7. S. Rausch, T. Binhammer, A. Harth, J. Kim, R. Ell, F. Kärtner, and U. Morgner, "Controlled waveforms on the single-cycle scale from a femtosecond oscillator," Opt. Express 16, 9739-9745 (2008).

8. S. A. Diddams, "The evolving optical frequency comb," J. Opt. Soc. Am. B 27, B51-B62 (2010).

9. S. Witte, R. Zinkstok, W. Hogervorst, and K. Eikema, "Control and precise measurement of carrier-envelope phase dynamics," Appl. Phys. B 78, 5-12 (2004).

10. L. Matos, O. D. Mücke, J. Chen, and F. X. Kärtner, "Carrier-envelope phase dynamics and noise analysis in octave-spanning Ti:sapphire lasers," Opt. Express 14, 2497-2511 (2006).

11. J. Reichert, R. Holzwarth, Th. Udem, and T. W. Hänsch, "Measuring the frequency of light with mode-locked lasers," Opt. Commun. 172, 59-68 (1999).

12. S. A. Diddams, D. J. Jones, J. Ye, S. T. Cundiff, J. L. Hall, J. K. Ranka, R. S. Windeler, R. Holzwarth, Th. Udem, and T. W. Hänsch, "Direct link between microwave and optical frequencies with a $300 \mathrm{THz}$ femtosecond laser comb," Phys. Rev. Lett. 84, 5102-5105 (2000).

13. D. J. Jones, S. A. Diddams, J. K. Ranka, A. Stentz, R. S. Windeler, J. L. Hall, and S. T. Cundiff, "Carrier-envelope phase control of femtosecond mode-locked lasers and direct optical frequency synthesis," Science 288, 635-639 (2000). 
14. G. Paulus, F. Lindner, H. Walther, A. Baltuska, E. Goulielmakis, M. Lezius, and F. Krausz, "Measurement of the phase of few-cycle laser pulses,” Phys. Rev. Lett. 91, 253004 (2003).

15. M. Krüger, M. Schenk, and P. Hommelhoff, "Attosecond control of electrons emitted from a nanoscale metal tip," Nature 475, 78-81 (2011).

16. A. Baltuska, Th. Udem, M. Uiberacker, M. Hentschel, E. Goulielmakis, Ch. Gohle, R. Holzwarth, V. S. Yakovlev, A. Scrinzi, T. W. Hänsch, and F. Krausz, "Attosecond control of electronic processes by intense light fields," Nature 421, 611-615 (2003).

17. L. Xu, C. Spielmann, A. Poppe, T. Brabec, F. Krausz, and T. W. Hänsch, "Route to phase control of ultrashort light pulses," Opt. Lett. 21, 2008-2010 (1996).

18. A. Poppe, R. Holzwarth, A. Apolonski, G. Tempea, C. Spielmann, T. W. Hänsch, and F. Krausz, "Few-cycle optical waveform synthesis," Appl. Phys. B 72, 373-376 (2001).

19. T. M. Fortier, J. Ye, S. T. Cundiff, and R. S. Windeler, "Nonlinear phase noise generated in air-silica microstructure fiber and its effect on carrier-envelope phase," Opt. Lett. 27, 445-447 (2002).

20. K. Holman, R. Jones, A. Marian, S. Cundiff, and J. Ye, "Detailed studies and control of intensity-related dynamics of femtosecond frequency combs from mode-locked ti:sapphire lasers," IEEE J. Sel. Top. Quantum Electron. 9, 1018-1024 (2003).

21. R. Paschotta, A. Schlatter, S. Zeller, H. Telle, and U. Keller, "Optical phase noise and carrier-envelope offset noise of mode-locked lasers," Appl. Phys. B 82, 265-273 (2006).

22. R. P. Scott, T. D. Mulder, K. A. Baker, and B. H. Kolner, "Amplitude and phase noise sensitivity of modelocked Ti:sapphire lasers in terms of a complex noise transfer function," Opt. Express 15, 9090-9095 (2007).

23. J. McFerran, W. Swann, B. Washburn, and N. Newbury, "Suppression of pump-induced frequency noise in fiberlaser frequency combs leading to sub-radian $f_{\text {ceo }}$ phase excursions," Appl. Phys. B 86, 219-227 (2007)

24. T. D. Mulder, R. P. Scott, and B. H. Kolner, "Amplitude and envelope phase noise of amodelocked laser predicted from its noise transfer function and the pumpnoise power spectrum,” Opt. Express 16, 14186-14191 (2008).

25. M. Y. Sander, E. P. Ippen, and F. X. Kärtner, "Carrier-envelope phase dynamics of octave-spanning dispersionmanaged Ti:sapphire lasers," Opt. Express 18, 4948-4960 (2010).

26. D. C. Heinecke, A. Bartels, and S. A. Diddams, "Offset frequency dynamics and phase noise properties of a self-referenced $10 \mathrm{GHz}$ Ti:sapphire frequency comb," Opt. Express 19, 18440-18451 (2011).

27. F. Helbing, G. Steinmeyer, and U. Keller, "Carrier-envelope offset phase-locking with attosecond timing jitter," IEEE J. Sel. Top. Quantum Electron. 9, 1030-1040 (2003).

\section{Introduction}

In the last decade, optical frequency combs have revolutionized ultrafast laser physics. They are now indispensable tools in a variety of fields ranging from optical frequency metrology [1] and optical clocks [2] to highly nonlinear optics and attosecond physics [3,4]. Among the several different femtosecond laser types that have been successfully employed as frequency combs, Ti:sapphire oscillators still play a very prominent role. The main reason is that they allow operation at the highest repetition rates [5], support the shortest pulses with octave-spanning spectra directly from the laser [6,7], and exhibit very low residual frequency noise due to the high quality factor of their resonators [8]. Nevertheless, from a practical and financial point of view, the main disadvantage of Ti:sapphire frequency combs when compared to fiber-based systems is the need for a bulky and costly pump laser emitting around $532 \mathrm{~nm}$ with excellent beam quality. For the most part, frequency-doubled single-frequency diode-pumped solid-state (DPSS) lasers have been used for this purpose. Detailed studies have shown underperformance with non-ideal pump lasers $[9,10]$. Recently, however, several cost-efficient alternative pump lasers with smaller footprints became commercially available. In this paper, we report on a characterization and comparison of the influence of four different commercial pump lasers on the carrier-envelope offset frequency stabilization of a femtosecond Ti:sapphire oscillator.

The remainder of this paper is organized as follows: After a review of the basics of carrierenvelope offset frequency stabilization and a short description of the tested pump lasers, the experimental setup and methods are introduced. Subsequently, the results of our measurements and their implications are presented and discussed. We find that all tested pump laser are wellsuited. 


\section{Basics}

It is well-known that the Fourier transform of the time-domain description of the infinite pulse train emitted from a mode-locked laser is given by a series of equidistant spectral lines whose frequencies $f_{n}$ are determined by $f_{n}=n f_{\mathrm{r}}+f_{0}$, where the mode number $n$ is an integer, $f_{\mathrm{r}}$ is the pulse repetition frequency, and $f_{0}$ denotes the carrier-envelope offset frequency. The latter is the time derivative of the carrier-envelope phase (CEP) $\phi_{0}$, which quantifies the phase advance of the electric carrier field with respect to the pulse envelope, and is given by

$$
f_{0}=\frac{1}{2 \pi} \frac{d \phi_{0}}{d t}=\frac{\Delta \phi_{0}}{2 \pi} f_{\mathrm{r}},
$$

where we have introduced the pulse-to-pulse slippage of the CEP $\Delta \phi_{0}$. Strictly speaking, it is not until both $f_{\mathrm{r}}$ and $f_{0}$ are measured and controlled that the spectrum of such a mode-locked laser forms a frequency comb [8]. While this can be routinely achieved for $f_{\mathrm{r}}$ using a fast photodiode and feedback to a piezo-eletric actuator changing the length of the laser resonator, the development and implementation of a scheme to measure $f_{0}$ was crucial for the realization of the first frequency combs $[11,12]$. The most commonly used method to access $f_{0}$ is the socalled $f$-to- $2 f$ interferometer, in which light at frequency $f$ from the low frequency part of the laser spectrum is frequency-doubled and subsequently heterodyned with light at frequency $2 f$ from the high frequency part. In general, the obvious requirement of an octave-spanning laser spectrum is challenging to be met directly even for Ti:sapphire oscillators, but can be overcome by nonlinear spectral broadening outside the laser, e.g. by self-phase modulation in a photonic crystal fiber (PCF) [13]. Once measured, stabilization of $f_{0}$ is possible by phase-locking $f_{0}$ to a stable rf reference and applying the generated feedback signal to the femtosecond oscillator.

Precise control of $f_{0}$ is not only required for frequency metrology, but is of even higher importance for extremely nonlinear time domain processes such as above-threshold ionization (ATI) $[14,15]$ and high harmonic generation (HHG) [16], whose outcome is critically determined by the CEP of the ultrashort laser pulses. However, since the measurement of $f_{0}$ is subject to noise and the servo-system controlling $f_{0}$ has a limited bandwidth, the stabilization of $f_{0}$ always suffers from residual noise, even for the ideal case of noiseless electronics. Therefore, the investigation of the different sources of noise that might impair the phase-locking of $f_{0}$ has been a long-standing and active field of research. In addition to rather obvious contributions such as mechanical and acoustic noise and imperfections of the feedback loop itself, several others were identified and analyzed both experimentally and theoretically by several research groups: It was shown that additional noise can result from fluctuations of the repetition rate of the oscillator, instabilities of the external spectral broadening in microstructured fibers, drifts in the second-harmonic power in the $f$-to- $2 f$ interferometer or fluctuations of pulse parameters that are not common mode for the frequencies $f$ and $2 f$ used for generating the $f_{0}$ beat note, and both beam pointing instabilities and intensity fluctuations of the pump laser $[9,10,17-26]$. The latter lead to intensity variations of the oscillator, which in turn cause phase variations due to amplitude-to-phase coupling via the nonlinear Kerr effect. While this enables active modulation of the amplitude of the pump laser as a means to apply feedback to the oscillator in the $f_{0}$ control loop, intrinsic intensity noise of the pump laser will limit the stabilization due to the same mechanism. Moreover, comparing a multi-longitudinal-mode and a single-mode pump laser, Witte et al. found that beam pointing fluctuations due to mode-fluctuations led to significantly higher phase noise [9]. Matos and co-workers also measured larger phase noise when pumping their octave-spanning Ti:sapphire frequency comb with a multi-mode pump laser. Based on a theoretical noise analysis of their system, however, they proposed that careful optimization of the feedback electronics should generally enable the same phase-locked performance as with a single-mode pump laser [10].

\#169150 - \$15.00 USD

(C) 2012 OSA
Received 23 May 2012; revised 3 Jul 2012; accepted 3 Jul 2012; published 26 Jul 2012 30 July 2012 / Vol. 20, No. 16 / OPTICS EXPRESS 18389 


\section{Devices under test}

The pump lasers tested in this set of experiments can be divided into two groups: The first group contains two frequency-doubled single frequency diode-pumped solid-state (DPSS) lasers emitting at $532 \mathrm{~nm}$ (Coherent Verdi V10, Coherent Verdi V5) that have been the pump lasers of choice for many years in applications with stringent requirements on phase stability due to their low intrinsic noise. The second group is formed by two lasers that run on many longitudinal modes: We used both a frequency-doubled DPSS laser with active noise cancellation (Lighthouse Photonics Sprout G-10W NET) and a frequency-doubled optically pumped semiconductor laser (OPSL, Coherent Verdi G5) that have become available as technologically advanced, small-sized, and cost-efficient alternatives to the lasers in the first group. The noise specifications of both multi-mode lasers $(<0.03 \%$ root-mean-square $(\mathrm{rms})$ from $10 \mathrm{~Hz}$ to $10 \mathrm{MHz}$ for the Sprout G-10W NET and $<0.02 \%$ rms from $10 \mathrm{~Hz}$ to $100 \mathrm{MHz}$ for the Verdi G5) are comparable to those of the single frequency lasers $(<0.02 \% \mathrm{rms}$ from $10 \mathrm{~Hz}$ to $1 \mathrm{GHz})$, thus making them promising candidates for pumping ultrafast lasers whose carrier-envelope offset frequency is to be stabilized.

To the best of our knowledge, we have contacted all manufacturers of suitable pump lasers of the latest generation prior to our experiments. The two multi-mode lasers were provided by the manufacturers on our request and did not seem to differ from a regularly sold model that we also tested. In contrast, both single-mode lasers have been used for CEP-critical experiments in our laboratories for many years. A potentially interesting third multi-mode laser of the latest generation (Laser Quantum Finesse) could not be provided at the time of our measurements by the manufacturer. Our efforts to test two additional multi- and single-mode DPSS lasers (SpectraPhysics Millennia Prime and Millennia Edge) that have been introduced to the market after completion of our initial measurements were insuccessful, because the manufacturer could not yet supply either model at that time. However, further studies are planned once these lasers and a designated low-noise version of the above-mentioned multi-mode laser (Finesse Pure) can be made available to us, and results will be published at a later point.

\section{Experimental setup and methods}

A schematic of our experimental setup is shown in Fig. 1. Pumped by the respective device under test at a power of $\sim 4.5 \mathrm{~W}$, a commercial Kerr-lens mode-locked Ti:sapphire oscillator (Femtolasers Femtosource 20) using chirped mirrors for dispersion management provides $\sim 20 \mathrm{fs}$ pulses at a repetition rate of $f_{\mathrm{r}}=102 \mathrm{MHz}$. We do not actively stabilize $f_{\mathrm{r}}$ throughout our experiments because the coupling between $f_{\mathrm{r}}$ and $f_{0}$ is negligible compared to other noise sources [27]. For stabilization of $f_{0}$, approximately $200 \mathrm{~mW}$ of the available oscillator output is sent to a nonlinear $f$-to- $2 f$ interferometer, which is located inside a box to minimize the effects of air flow and acoustic noise. First, the oscillator output spectrum $(\sim 40 \mathrm{~nm}$ FWHM $)$ is spectrally broadened to support a full optical octave in a $60 \mathrm{~mm}$ long, angle-polished, endsealed PCF (MenloSystems) with a core diameter of $1.9 \mu \mathrm{m}$ and a zero-dispersion wavelength of $810 \mathrm{~nm}$. A dichroic mirror then splits the infrared and the blue part of the spectrum. The former is frequency-doubled in a $5 \mathrm{~mm}$ long $\mathrm{KNbO}_{3}$ crystal and subsequently overlapped spatially and temporally with the latter by means of a polarizing beam splitter and a variable delay stage. After mapping both beams onto a common polarization axis, the heterodyne signal is isolated with a grating (2100lines/ $\mathrm{mm}$ ) and sent to an avalanche photo diode (APD, MenloSystems APD 210). The signal from the APD is processed and compared to a reference signal at $21.25 \mathrm{MHz}$ provided by an rf function generator (Stanford Research Systems DS345). The so generated feedback signal is applied to an acousto-optic modulator (AOM, IntraAction AFM 405A1), which modulates $f_{0}$ via the pump laser power sent to the Ti:sapphire oscillator. We use two internally different versions of commercially available electronics (MenloSystems

\#169150 - \$15.00 USD

(C) 2012 OSA
Received 23 May 2012; revised 3 Jul 2012; accepted 3 Jul 2012; published 26 Jul 2012 30 July 2012 / Vol. 20, No. 16 / OPTICS EXPRESS 18390 


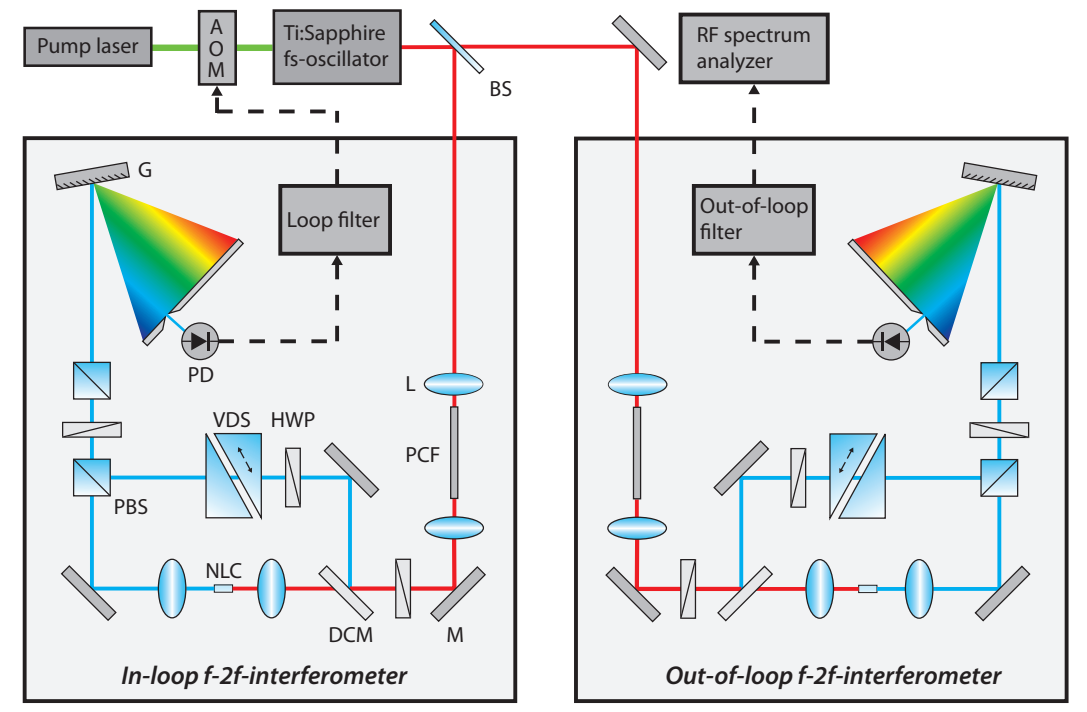

Fig. 1. Schematic of experimental setup. AOM: acousto-optic modulator, BS: beam splitter, L: lens, PCF: photonic crystal fiber, M: mirror, HWP: half-wave plate, DCM: dichroic mirror, NLC: nonlinear crystal, VDS: variable delay stage, PBS: polarizing beam splitter, G: grating, PD: photodiode, (Out-of-)Loop filter: MenloSystems XPS800-E. See text for details.

XPS800-E) as loop filters for signal processing and locking.

To measure the residual phase noise of the $f_{0}$-stabilized oscillator, the remaining part of the oscillator output $(\sim 200 \mathrm{~mW})$ is sent to a second, equally shielded $f$-to- $2 f$ interferometer that is not part of the feedback loop (and thus also termed out-of-loop interferometer in the following). The signal acqusition is performed in two ways: In the frequency domain, we use an $\mathrm{rf}$ spectrum analyzer (Agilent E4445A) to obtain the spectrum of the $f_{0}$ beat signal recorded with the APD for different frequency spans and resolution bandwidths. Using commercial software (MenloSystems PhaseNoise), each of these datasets is normalized to the respective resolution bandwidth and to the power of the beat note signal $f_{0}$ and then stitched together to obtain the phase noise power spectral density, from which the total residual phase noise can be calculated via integration from high frequencies to low frequencies. A complementary time domain measurement is performed directly with a personal computer. After digitizing the heterodyne signal from the phase detector with a data acquisition module (National Instruments USB6251), the voltage data is recorded and processed by the above-mentioned software. From the phase and timing jitter calculated from the data, the phase noise power spectral density and thus the residual phase noise is obtained via Fourier transformation and integration. Using these two different methods, the residual phase noise is measured both with the in-loop and the out-ofloop interferometer and both available locking electronics in all possible combinations.

In order to be able to assess the potential influence of the intensity fluctuations of the pump lasers on the stabilization of $f_{0}$, a small portion of the pump laser beam (either split from the main beam with a wedge or leaking through one of the pump mirrors) was focused onto a 9 Volt biased PIN diode (Hamamatsu S5973). The signal from the photodiode was sent to a personal computer using the same data acquisition card as in the phase noise measurements and recorded by means of the same software. This enabled us to characterize the relative intensity noise (RIN) of all four pump lasers in the range between $2 \mathrm{~Hz}$ and $625 \mathrm{kHz}$. 

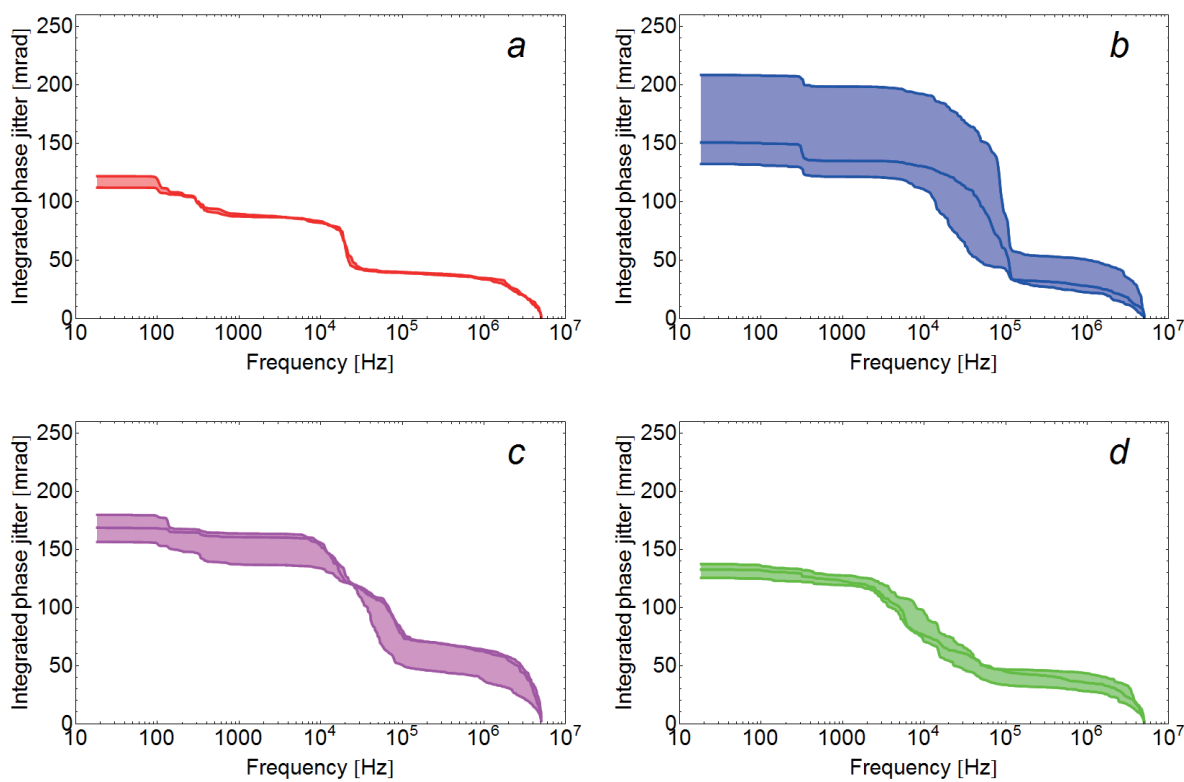

Fig. 2. Comparison of integrated carrier-envelope offset phase jitter datasets of a) V10, b) V5, c) G5, and d) Sprout. Note that the scale is the same in all plots and that the number of measured datasets is different for the V10.

\section{Results and discussion}

\subsection{Phase noise of stabilized carrier-envelope frequency}

Figure 2 summarizes the results of our phase noise measurements of the stabilized carrier envelope frequency. It shows the respective frequency-dependent rms integrated phase jitter $\Delta \phi_{0}$ of our Ti:sapphire oscillator when pumped by each of the four tested pump lasers. For each laser, the noise band formed by multiple measurements is indicated. The data shown here were obtained from the out-of-loop interferometer with the rf spectrum analyzer. We verified that our measurements were not limited by the rf spectrum analyzer in the frequeny region of interest (i.e., significantly contributing to the PSD). However, as we will show in this section (see Fig. 4), the noise floor depends on the signal-to-noise ratio $(\mathrm{S} / \mathrm{N})$ of the offset beat note. We also confirmed that the results obtained using the time domain method described above agree well with the data shown in Fig. 2. Since the depicted results include the noise of both electronics setups and both interferometers, they represent an upper limit of the achievable performance of the stabilization scheme and, thus, the stability of $f_{0}$ when using the different pump lasers.

Apart from comparing the total phase noise, it is interesting to analyze at which frequencies in the experimentally accessible range $(20 \mathrm{~Hz}-5 \mathrm{MHz}$ in our measurements) there are significant contributions to the integrated phase noise. In the following discussion, we will thus concentrate on the changes in integrated phase noise with frequency for each of the pump lasers. In general, phase fluctuations at high frequencies are particularly important because noise at lower frequencies can be suppressed rather well by a standard feedback loop with a bandwidth reaching several $10 \mathrm{kHz}$. At the same time, contributions at frequencies above the frequency of the relaxation oscillations of the Ti:sapphire oscillator (usually several $100 \mathrm{kHz}$ ) are effectively filtered because of the long lifetime of the upper laser level [22]. It can be seen from Fig. 2 that the frequency range between $10 \mathrm{kHz}$ and $100 \mathrm{kHz}$ significantly contributes to the phase noise

$\# 169150$ - \$15.00 USD

(C) 2012 OSA 

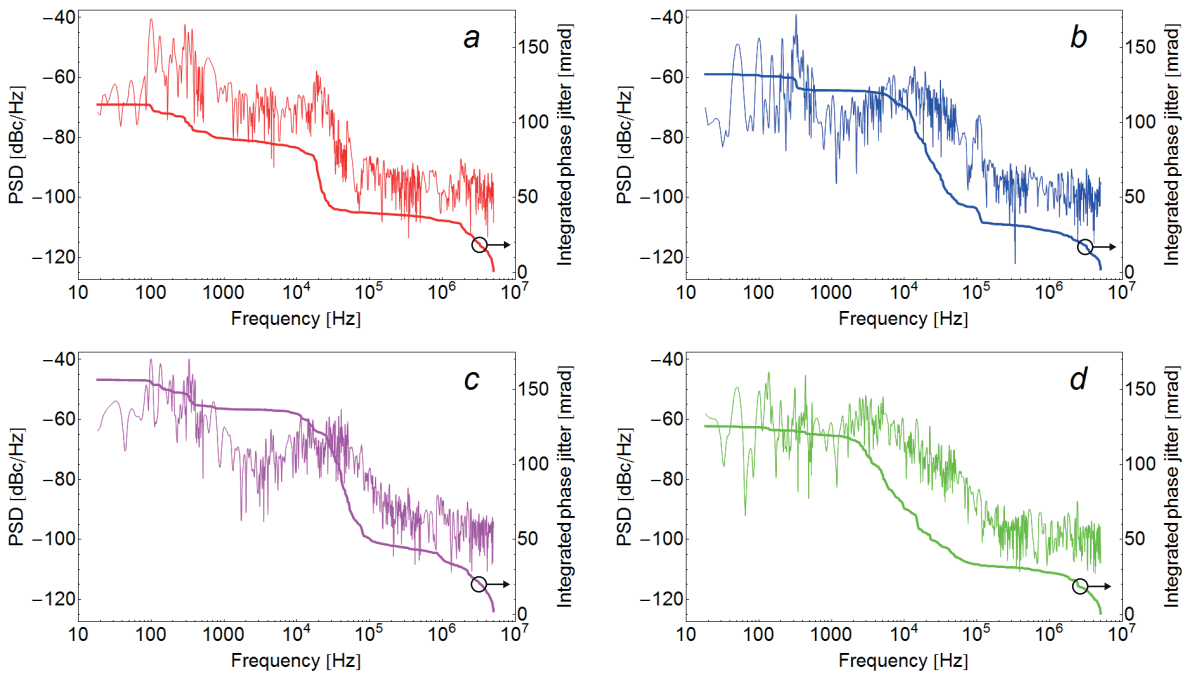

Fig. 3. Comparison of minimum integrated phase jitter (thick lines) and underlying power spectral density (PSD, thin lines) obtained with the tested pump lasers in the range between $20 \mathrm{~Hz}$ and $5 \mathrm{MHz}$. For all pump lasers, the residual phase noise stays below 1/40 of an optical cycle. The obtained values are a) $112 \mathrm{mrad}$ for the V10, b) $132 \mathrm{mrad}$ for the V5, c) $156 \mathrm{mrad}$ for the G5, and d) $126 \mathrm{mrad}$ for the Sprout. The main phase noise contributions originate from the frequency interval $10-100 \mathrm{kHz}$ for the Verdi pump lasers (V10, V5, G5) and from the somewhat larger $1-100 \mathrm{kHz}$ range for the Sprout.

for all pump lasers. The effect is most prominent for the Verdi V5 (Fig. 2(b)) and least pronounced for the Verdi V10 (Fig. 2(a)). For both the latter and the Sprout, the phase jitter does not exceed $100 \mathrm{mrad}$ for frequencies above $10 \mathrm{kHz}$ in all our measurements. When comparing the repeatability of our measurements, the V5 clearly shows the largest scatter among different measurements which results in an increased width of the noise band in Fig. 2.

However, it is important to make the point that we found all four pump lasers to be wellsuited for stabilization of $f_{0}$. In Fig. 3, we show the power spectral density and the integrated phase noise that correspond to the best phase noise performance achieved in our experiments for each of the four tested pump lasers. The minimum integrated phase jitter is $112 \mathrm{mrad}$ for the V10 (Fig. 3(a)), $132 \mathrm{mrad}$ for the V5 (Fig. 3(b)), $156 \mathrm{mrad}$ for the G5 (Fig. 3(c)), and $126 \mathrm{mrad}$ for the Sprout (Fig. 3(d)) in the range between $20 \mathrm{~Hz}$ and $5 \mathrm{MHz}$. These values correspond to less than $1 / 40$ of an optical period for all pump lasers and can be converted into an rms timing jitter of 48 as for the V10, 57 as for the V5, 65 as for the G5, and 54 as for the Sprout. In contrast to the results reported in [9], we cannot find a systematic difference in phase noise performance depending on the use of either the single-mode or two multi-mode lasers.

It is instructive to take a closer look on the influence of the signal-to-noise ratio $(\mathrm{S} / \mathrm{N})$ of the beat note $f_{0}$ on the measured phase jitter. We exemplify this effect in Fig. 4 with two datasets for which the integrated phase noise differs by almost a factor of 2 although the identical pump laser (V10) has been used in both cases. The main part of the difference in phase noise is accumulated at frequencies above $1 \mathrm{MHz}$ and thus outside the range of the servo loop. To illustrate the influence of the $\mathrm{S} / \mathrm{N}$, the rf spectra of the beat note, from which the phase noise in that frequency range has been calculated (10 MHz span, $91 \mathrm{kHz}$ resolution bandwidth), are shown in Fig. 4(b). As can be seen, the $\mathrm{S} / \mathrm{N}$ is about $30 \mathrm{~dB}$ for the noisier curve (dashed, black) and about $40 \mathrm{~dB}$ for the other measurement (solid, blue) while the background is similarly

\#169150 - \$15.00 USD

(C) 2012 OSA
Received 23 May 2012; revised 3 Jul 2012; accepted 3 Jul 2012; published 26 Jul 2012

30 July 2012 / Vol. 20, No. 16 / OPTICS EXPRESS 18393 

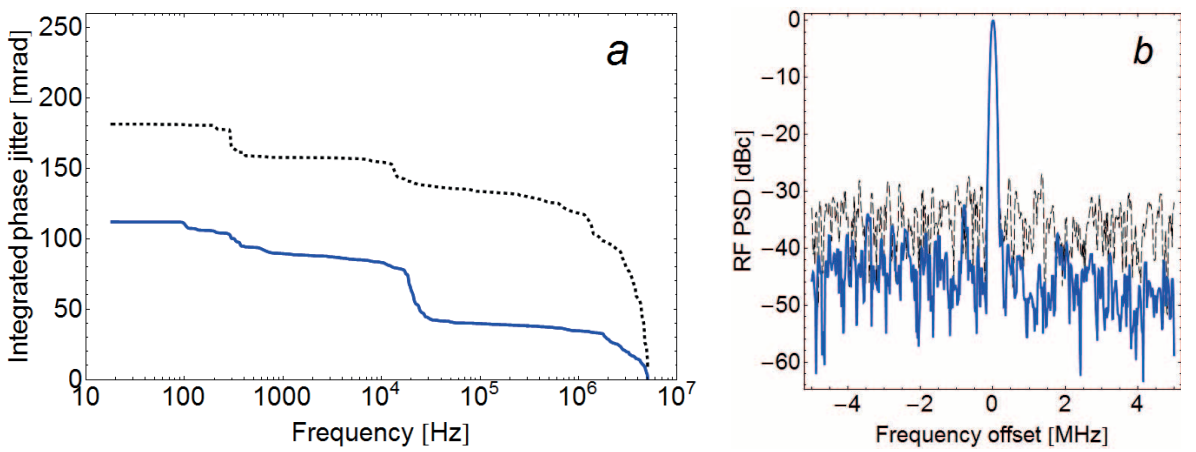

Fig. 4. Comparison of influence of signal-to-noise ratio on phase noise exemplified by two different datasets obtained with the Verdi V10 and the frequency domain method. The dataset resulting in lower phase noise (solid blue line) was recorded with $10 \mathrm{~dB}$ more $\mathrm{S} / \mathrm{N}$ than the other one (dashed black line) in the frequency range above $1 \mathrm{MHz}$, where the main difference in phase noise is accumulated. This can be seen in the corresponding rf spectra (10 MHz span, $91 \mathrm{kHz}$ resolution bandwidth) shown on the right.

well-behaved for both datasets. The resulting phase noise accumulated above $1 \mathrm{MHz}$ differs by almost $100 \mathrm{mrad}$. Therefore, as a rule of thumb, we suggest that the $\mathrm{S} / \mathrm{N}$ should be at least better than $35 \mathrm{~dB}$ for $\sim 100 \mathrm{kHz}$ bandwidth to obtain meaningful data, because the real signal may be obscured by the background noise otherwise. Consequently, all phase noise data shown in this paper were selected according to this rule.

\subsection{Relative intensity noise}

The integrated relative intensity noise (RIN) of each of the four pump lasers is shown in Fig. 5. Different line styles and colors indicate datasets from different days. The RIN data from the respective day of the phase noise measurement is shown as solid (blue) lines for each laser. The power levels the data were taken at were $3 \mathrm{~W}$ for the $\mathrm{V} 10,3 \mathrm{~W}$ for the $\mathrm{V} 5,4.8 \mathrm{~W}$ for the $\mathrm{G} 5$, and $3 \mathrm{~W}$ for the Sprout. Since operation at higher output power typically yields lower RIN, we took our RIN data at or not too far below the power levels used for pumping the Ti:sapphire oscillator during the phase noise measurements. Note that our experiments covered the frequency interval $2 \mathrm{~Hz}-625 \mathrm{kHz}$ so that the results include intensity noise at lower frequencies than specified by the manufacturers while at the same time not resolving the high frequency part of the specified range. This has to be taken into account when comparing measured and specified RIN.

In our experiments, both single-frequency lasers (top row in Fig. 5) show pronounced intensity fluctuations around $100 \mathrm{kHz}$. For the V5 (top right), the noise feature is much stronger and constitutes a major contribution to the RIN, whereas it is is less distinct for the V10 (top left), which in contrast exhibits a considerable amount of RIN below $600 \mathrm{~Hz}$. Interestingly, our V10 reveals additional noise contributions below $10 \mathrm{~Hz}$ that are not present for the V5. The large day-to-day scatter in the RIN data of the latter triggered a set of independent measurements in which we observed a similarly fluctuating behavior of the RIN within the same day. While the origin of these time-dependent fluctuations remains unclear to us, a possible explanation for the generally declined performance of the single-frequency lasers compared to the specifications of the manufacturer could simply be aging. As mentioned before, both the V10 and the V5 have been in extensive use in our laboratories for several years. Remarkably, as can be seen in Fig. 2, the intensity noise around $100 \mathrm{kHz}$ does not seem to translate into phase noise

\#169150 - \$15.00 USD

(C) 2012 OSA
Received 23 May 2012; revised 3 Jul 2012; accepted 3 Jul 2012; published 26 Jul 2012

30 July 2012 / Vol. 20, No. 16 / OPTICS EXPRESS 18394 

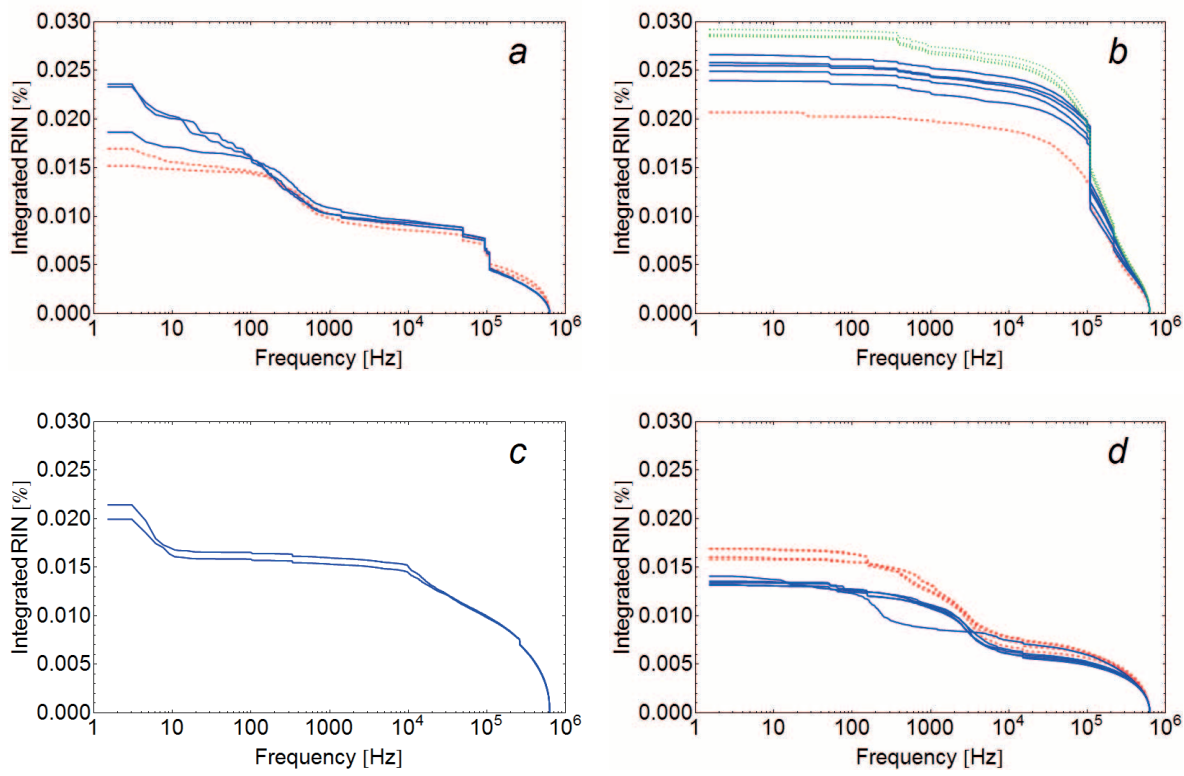

Fig. 5. Comparison of integrated relative intensity noise of a) V10, b) V5, c) G5, and d) Sprout in the frequency range between $2 \mathrm{~Hz}$ and $625 \mathrm{kHz}$. Different line styles (colors) indicate datasets from different days. The RIN data from the respective day of the phase noise measurement is shown as solid (blue) lines. Note that the RIN was measured at slightly different power levels for different pump lasers and that the number of recorded datasets is different for each laser.

for the V10, whereas it coincides with the steep rise in the upper bound of the phase noise band of the V5. While we cannot identify further direct influence of the RIN on the phase noise performance for the latter, the increased intensity fluctuations of the V10 at frequencies below $\sim 600 \mathrm{~Hz}$ might be the reason for the observable contribution to the residual phase jitter at these low frequencies. However, a better adjusted loop filter should generally be able to cancel this noise.

Both multi-mode lasers (bottom row in Fig. 5) show a comparably low and smooth integrated RIN. The Verdi G5 (Fig. 5(c)) accumulates the majority of its RIN above $10 \mathrm{kHz}$ and below $10 \mathrm{~Hz}$. The Sprout (Fig. 5(d)) exhibits the lowest RIN of all tested pump lasers. We confirmed the low RIN of the Sprout models with active noise cancellation in additional tests with two more lasers of the same type (Sprout G-10W NET and G-6W NET). The most significant intensity fluctuations of the Sprout can be observed at frequencies from $100 \mathrm{~Hz}$ to $1 \mathrm{kHz}$. However, the interval where the main phase jitter is accumulated (2-60kHz, compare Fig. 2) cannot be unambiguously linked to these intensity fluctuations. For the G5, the largest contribution in phase noise lies between $10 \mathrm{kHz}$ and $100 \mathrm{kHz}$ and thus in a span where we also observe strong intensity noise. Yet, looking at Fig. 3, it becomes obvious that all lasers share the large increase in accumulated phase jitter in the same frequency range $(10-100 \mathrm{kHz})$ irrespective of their RIN behavior, which leads us to the conclusion that it is rather caused by the limited bandwidth of our servo system. 


\section{Summary}

Using four different commercially available single- and multi-longitudinal-mode pump lasers, we have stabilized the carrier-envelope frequency $f_{0}$ of a Ti:sapphire femtosecond oscillator by means of the $f$-to- $2 f$ self-referencing technique. The residual phase noise of the stabilized femtosecond laser was measured with a second identical $f$-to- $2 f$ interferometer outside the feedback loop. In order to analyze the influence of intensity fluctuations of the pump lasers on the stabilization, we also characterized the relative intensity noise of the pump lasers.

We found all pump lasers under test to be suitable for carrier-envelope frequency stabilization, irrespective of their single- or multi-mode operation. The best performance of the oscillator obtained with each pump laser corresponded to an integrated rms residual phase jitter of below $160 \mathrm{mrad}$, which is less than $1 / 40$ of an optical cycle and amounts to an rms timing jitter of less than 70 as. We observed different repeatabilities in phase noise performance of the oscillator for different pump lasers, yet far from hampering stabilization.

The relative intensity noise of the pump lasers was measured and compared to the residual phase noise of the oscillator when pumped by the respective pump laser. While certain noise features of the pump sources can be seen to directly translate into phase noise of the oscillator, pumping the oscillator with the quietest source did not yield the lowest overall residual phase noise. None of the tested lasers showed intensity fluctuations strong enough to prevent stabilization of $f_{0}$.

$\# 169150$ - \$15.00 USD

(C) 2012 OSA
Received 23 May 2012; revised 3 Jul 2012; accepted 3 Jul 2012; published 26 Jul 2012 30 July 2012 / Vol. 20, No. 16 / OPTICS EXPRESS 18396 